\title{
Conflitos e abordagem bioética de crianças com distúrbios do desenvolvimento sexual
}

\author{
Conflicts and bioethical approach of children born \\ with disorders of sexual development
}

\begin{abstract}
Ida Cristina Gubert
Doutora em Imunologia e Farmacologia Bioquímica e Molecular Universidade Federal de Minas Gerais (UFMG), ph.D em Ética e Investigação Clínica pela Faculdade Latino Americana de Ciências Sociais (FLACSO), Buenos Aires - Argentina, e-mail: gubert@ufpr.br
\end{abstract}

\section{Resumo}

Hermafroditismo, genitália ambígua, intersexo são termos hoje sob a designação de distúrbios do desenvolvimento sexual e que traduzem, em última análise, uma discordância entre o sexo cariotípico, fenotípico e gonadal, resultando em uma indefinição ou ambiguidade da sexualidade da criança afetada. Essas condições, ainda que raras, e até pouco tempo não muito divulgadas em função do estigma e da vergonha, hoje estão mais abertas e difundidas, mas sua abordagem ainda é controversa. Aspectos desafiantes da abordagem e conduta para com os sujeitos afetados pelos distúrbios do desenvolvimento sexual são a pressão da sociedade pelo conhecimento e consequentemente definição do sexo da criança, a autonomia desse sujeito, os princípios de beneficência, a tomada de decisões "no melhor interesse da criança" e o consentimento/ 
assentimento para a abordagem terapêutica mais adequada. As controvérsias expostas no texto convidam a uma reflexão multidisciplinar no enfoque desses casos.

Palavras-chave: Bioética. Intersexo. Ambiguidade genital. Distúrbios do desenvolvimento sexual.

\section{Abstract}

Disorders of sexual development is the term now used to encompass hermaphroditism, ambiguous genitalia and intersex, conditions in which there is a divergence between chromossomal and phenotypic sex resulting in sexual ambiguity or indefinition for the child affected. These rather rare conditions, although long known by geneticists and clinicians, that have for a long time kept in secrecy due to shame and stigma, are now more openly talked about, but their approach is still controversial. Challenging aspects in the approach and conduction unto the subjects affected are the pressure from society in wishing to know the baby's sex and consequently its definition, the subject's autonomy, the basic principles of beneficence, decision making in "the best interest of the child" and consent/assent for the most adequate approach and treatment of these conditions. Controversial topics are presented in the text, inviting us to a multidisciplinary reflection on these cases.

Keywords: Bioethics. Intersex. Ambiguous genitalia. Disorders of sexual development.

\section{Introdução}

A genética sempre se apresentou como uma ciência desafiante dada à amplitude de seu espectro na relação causal, ou relacional, com determinadas condições da saúde humana. A cada nova investida no genoma humano, etapas na atividade ou função do gene são identificadas e relações causais são atribuídas a algumas doenças, antes de origem desconhecida. Contudo, há que se tomar cuidado, pois não podemos cair no determinismo ou reducionismo, já que nem tudo é responsabilidade da genética e são de fundamental importância as interações com o microambiente interno e externo ao organismo humano. Embora os distúrbios do 
desenvolvimento sexual (DDS), de fato, tenham uma relação importante com a genética, não se pretende, com este texto, analisar em detalhes cada distúrbio. Pretende-se, sim, tecer uma abordagem bioética dessas condições, já que as implicações são de extrema importância para as crianças afetadas, para seus pais e para os profissionais da saúde que os atendem.

\section{Os distúrbos do desenvolvimento sexual}

A curiosidade pelo sexo dos filhos sempre esteve presente na mente dos futuros pais, e os avanços nos recursos para análises genéticas tais como amniocentese e cariotipagem, primeiramente, seguidos da ultrassonografia permitem identificar com bastante segurança o sexo do bebê desde muito precocemente na gestação; assim como, as técnicas de fertilização assistida, utilizando as metodologias atualmente disponíveis, permitem a seleção dos embriões antes da implantação. A algumas famílias vêm utilizando esses recursos para escolher um bebê deste ou daquele sexo.

Todos nós, seres humanos, possuímos um conjunto de 23 pares cromossômicos, que nada mais são do que pequenos filamentos nos quais se encontra organizada nossa informação genética. Desses 23 pares, 22 são conhecidos como autossômicos e um par corresponde aos cromossomos sexuais (XX ou XY). Quando da divisão celular, para a distribuição dos cromossomos no momento da concepção, algumas vezes ocorrem desarranjos que fogem ao nosso controle, e que podem resultar em perda ou ganho de um cromossomo ou de um segmento cromossômico, resultando em uma alteração na "conformação" da pessoa. Assim, podem ocorrer ganhos de cromossomo $\mathrm{Y}$ e o ser em formação terá um cariótipo XYY, mas pode haver ganho de cromossomo X - XXY, XXX ou ainda perda desse cromossomo quando então a criança será apenas $X$. A gravidade dessas anomalias é variável, mas a infertilidade é um dado presente (PASTERNACK, 2002).

Entretanto, a mera presença dos cromossomos sexuais pode não ser suficiente para a determinação sexual funcional dentro dos parâmetros estabelecidos como masculino e feminino. As interações com o meio ambiente interno e, compreenda-se aí, hormônios e enzimas, vão contribuir para a diferenciação sexual secundária e a determinação efetiva 
do sexo. Assim, alguns casos de distúrbios do desenvolvimento sexual podem até apresentar o conjunto cromossômico correto, mas as interações com os hormônios e enzimas, ou sua inexistência, podem desviar da normalidade o sexo da criança. Ainda, pode haver condições de agenesia/ disgenesia gonadal, ou seja, gônadas ausentes ou reduzidas, ambiguidade genital, que também comprometerão a função sexual. Uma classificação extensa e detalhada dos distúrbios do desenvolvimento sexual abordando as alterações cromossômicas, as má-formações de gônadas bem como a ação dos diversos hormônios sobre as estruturas sexuais da criança em desenvolvimento, dentre outras alterações que resultam em DDS, e suas consequências, e a melhor abordagem dessas condições, podem ser encontrados em Barbaro, Wedell e Nordenström (2011), Barthold (2011), Hughes et al. (2006, 2007) e Rey e Grinspon (2011).

\section{O sujeito e a sociedade}

Durante muito tempo, os indivíduos afetados por distúrbios do desenvolvimento sexual não manifestavam sua condição pelas mais diversas razões. Hoje, existem associações bem organizadas, como a ISNA (Intersexed Society of North America), fundada por Cheryl Chase (MARTIN, 2002), que proporcionam a essas pessoas aconselhamento, troca de informações e ideias; e, com isso, os casos de DDS, conhecidos há bastante tempo pelos mais familiarizados com a genética, na atualidade recebem uma nomenclatura menos estigmatizante e mais abrangente. Assim, termos como hermafroditismo, intersexo e genitália ambígua, para citar alguns, hoje estão incluídos como distúrbios do desenvolvimento sexual (DDS) (ALLEN, 2009).

Uma das características dos DDSs é justamente a indefinição ou a ambiguidade na definição do sexo, fugindo do sistema binário estabelecido, de sexo ou gênero masculino ou feminino. De hermafroditas, para intersexo, para transgêneros, buscamos a identidade sexual daqueles que ultrapassaram os limites do masculino/feminino (MARTIN, 2002).

Fausto-Sterling (1993 apud MARTIN, 2002) então defende a existência de cinco sexos enquanto Greenberg (1999 apud MARTIN, 2002) trabalha gênero, sexo, identidade sexual e orientação sexual do ponto de vista legal. 
Entretanto há que se diferenciar o que seja efetivamente distúrbio do desenvolvimento sexual das situações que resultam da preferência individual do sujeito por esse ou aquele sexo ou ainda dos casos de acidentes durante o processo de circuncisão.

\section{Diagnóstico}

O diagnóstico dos DDSs é dificultado por uma série de fatores dentre os quais podem ser citados as diferentes metodologias empregadas pelos diferentes grupos, o pequeno tamanho das amostras, a inexistência de grupos controle e de estudos de longo termo (FRADER, 2004; LUX, 2009).

Em função da importância do quadro, o diagnóstico de DDS deve envolver levantamento da história (anamnese), exame físico e investigações laboratoriais, como cariótipo, por exemplo, dentre outras, e pode ser genético, cromossômico, endocrinológico, clínico e por imagem, mas a cariotipagem é fundamental para todos os casos de DDS (ALLEN, 2009).

\section{Reatribuição de sexo}

Os DDSs traduzem condições em que há conflito ou discordância entre sexo cromossômico e gonadal, podendo diferir do padrão estabelecido como masculino e feminino. A reatribuição de sexo tem a finalidade de buscar minimizar as dificuldades decorrentes de distúrbios do desenvolvimento sexual. São exemplos: a hiperplasia adrenal congênita (CAA), que produz virilização do feto feminino, em graus variáveis; a síndrome da insensibilidade androgênica (AIS), que produz indivíduos 46XY com testículos intrabdominais ou contidos em hérnia inguinal e sem genitália interna feminina; meninos 46XY gravemente não virilizados e, por fim, os casos de hermafroditismo verdadeiro/disgenesia gonadal mista (RANGECROFT, 2003); Barbaro, Wedell e Nordenström (2011), Barthold (2011), Hughes et al. (2006, 2007) e Rey e Grinspon (2011).

Diferentemente dos casos de DDS de etiologia genética ou cromossômica, Bradley et al. (1998) descrevem dois casos emblemáticos de 
reatribuição de sexo em meninos, com sexo cromossômico $\mathrm{XY}$, que sofreram ablação do pênis em um acidente durante o processo de circuncisão. Após a castração cirúrgica e a reconstrução genital, os sujeitos foram submetidos à terapia feminilizante. Os resultados desse processo são distintos. No primeiro caso, a pessoa se suicidou como resultado da não adaptação ao sexo reatribuído; no segundo caso, a pessoa vivia em conformidade com a sua condição feminina. Em ambos os casos realizou-se cirurgia estética e procedeu-se uma modificação das características sexuais secundárias a partir da terapia de reposição hormonal.

Money tratou um caso emblemático de reatribuição de sexo de um menino que teve o pênis decepado no momento da circuncisão (MONEY, 1975 apud ALLEN, 2009). O nome verdadeiro, David Reimer, foi revelado tempos depois, em livro e entrevista de David a Colapinto (MARTIN, 2002). David Reimer suicidou-se aos 34 anos, depois de viver em conflito com sua sexualidade (WALKER, 2004).

John Money, psicólogo estudioso da sexualidade no Hospital John Hopkins, postulava a neutralidade sexual da criança até os 2 anos de idade; ainda, tinha como princípio que a cirurgia feminilizante, aliada à terapia com hormônios feminilizantes e mais o envolvimento afetivo da família resultavam na aceitação final do sentido do gênero conforme atribuído. Para ele, o sexo de criação era o determinante mais importante da identidade de gênero de uma pessoa (MONEY, 1975 apud ALLEN, 2009).

No entanto, ao longo dos anos, com os resultados desastrosos desse conceito sendo revelados e as teorias opostas apresentadas por Diamond (1965), o argumento nature versus nurture (natureza vs criação) de Money (1975 apud ALLEN, 2009) foi caindo no esquecimento. Importante ressaltar que à época, a prática médica era essencialmente hipocrática e não levava em conta a autonomia do sujeito; assim, o médico decidia com caráter de urgência os procedimentos a serem adotados para as crianças com DDS, e pais e crianças afetadas recebiam pouca ou nenhuma informação a respeito da condição. Atualmente, o padrão de atendimento consiste em informar aos pais, discutir as opções e dar a conhecer, de maneira progressiva e adequada à sua idade, às crianças sobre sua condição (ALLEN, 2009; MIESZCZAK, 2009). 
Cariótipo, função gonadal, fenótipo, características da genitália interna, riscos de malignidade, de disforia (insatisfação) com o gênero atribuído, são alguns dos aspectos relevantes a serem considerados quando da reatribuição de sexo nos casos de DDS e descritos por Allen (2009).

\section{Abordagem bioética}

Uma vez firmado o diagnóstico, o próximo passo é a revelação da problemática e sua abordagem; nesse momento, é importante auxiliar a família a compreender corretamente a condição da criança; contar para os bebês a sua história ainda na fase de recém-nascidos e fornecer respostas honestas às crianças, mas de forma adequada a seu nível de compreensão (ALLEN, 2009).

Kipnis (2004), em seu artigo, diz que os médicos não devem mentir, pois a mentira denigre a arte da medicina e que a beneficência deve ser considerada como uma aspiração ética distintiva e a maleficência como uma traição culpável desse valor e não uma falha em realizar. Assim como, as deliberações do grupo multidisciplinar apoiado pelo The Hastings Center apontam para a necessidade da revelação da verdade às crianças (FRADER et al., 2004).

A multidisciplinaridade é mandatória na abordagem do sujeito DDS (ALLEN, 2009; FRADER, 2004; HUGHES, 2006; MAHARAJ et al., 2005; RANGECROFT, 2003) e deve, na medida do possível, incluir endocrinologista, urologista/cirurgião, psicólogo/psiquiatra, geneticista, neonatologista e ginecologista, além de assistente social, corpo de enfermagem e bioeticista (BARTHOLD, 2011). Gillam (2010) apresentam diretrizes éticas para a abordagem da criança DDS consistentes com os princípios de Rawls, do equilíbrio reflexivo. Apontam também para a minimização dos riscos, o respeito aos desejos e crenças dos pais, e a opção de um futuro aberto para a criança como a melhor conduta para minorar as dificuldades enfrentadas por essas pessoas.

A atribuição de gênero e os cuidados com esses sujeitos devem abranger equipes multidisciplinares para atender os desafios médicos, cirúrgicos e psicossociais (ALLEN, 2009). Também devem levar em 
consideração as diferenças entre as cirurgias, considerando-se as emergências médicas e as cirurgias meramente estéticas. A condição DDS é conflituosa em sua essência e, mais ainda, para as relações profissionais-crianças-famílias. Dois tipos de comportamento podem ser encontrados nessa relação pais-profissionais: a demanda informada, em que os pais querem que os médicos "consertem" seu filho, ou a recusa informada, que introduz aspectos complicados de negligência (KIPNIS, 2004).

Kipnis (2003) apresenta sete tipos de vulnerabilidades a que estão expostas as crianças, em um contexto de pesquisa, mas que se aplicam também às condições DDS. Um dos aspectos relevantes e prementes nos casos de DDS é a cirurgia corretiva. Nesse tema, encontram-se grandes conflitos e dilemas, abordados na literatura, e cuja preconização foi sendo modificada na medida da mudança de paradigmas (DAABOUL; FRADER, 2001) no atendimento a esses sujeitos, passando do modelo tecno-centrado e paternalista, para o modelo centrado no paciente, bem como do posicionamento dos sujeitos DDS com relação à sua condição (DREGER, 1999).

Embora Daaboul e Frader (2001) se posicionem a favor de mudanças na conduta a ser adotada com os sujeitos DDS, fugindo do sistema paternalista, também tecem considerações sobre os riscos de malefícios que podem resultar da demora na realização das cirurgias, conforme defendido por alguns sujeitos com DDS (DREGER, 1999) e propõem um meio termo na tomada de decisões.

\section{Confidencialidade e vulnerabilidade}

A confidencialidade se constitui como um aspecto delicado da relação profissional da saúde e seu paciente, e não menos importante no caso dos DDS. Além da criança afetada pelo DDS ter o direito de saber, conhecer e compreender sua condição, o apoio familiar pode minimizar a vulnerabilidade da criança. Se por um lado as crianças podem sentir-se tolhidas na presença dos pais para revelar ou discutir questões inerentes às suas intimidades, por outro lado, o respeito à confidencialidade e a privacidade na relação médico-paciente pode contribuir para aumentar a 
confiança da criança no profissional que a atende (HUGHES et al., 2007; KIPNIS, 2004). No entanto a quebra de confidencialidade pode ser justificada nos casos em que há necessidade de proteger a criança de um dano maior (KIPNIS, 2004).

Hughes et al. (2007) comentam, por exemplo, que os exames clínicos repetidos, mais a obtenção de fotos, algumas vezes efetivamente necessárias, são fatores que traumatizam o sujeito DDS, contribuindo para a vulnerabilidade. A premência em corrigir a condição tem justificativa justamente na vulnerabilidade do sujeito. No entanto decisões tomadas por outrem, ou mesmo pela criança, se ainda não totalmente madura para tal, podem gerar problemas de disforia de gênero (ALLEN, 2009), dificuldades de ordem psicológica, levando à depressão e eventualmente ao suicídio. Por outro lado, não é apenas a criança portadora de DDS que apresenta vulnerabilidades. O profissional da saúde também pode ser defrontado com uma vulnerabilidade e com processos judiciais mediante a insatisfação do sujeito DDS com o resultado da cirurgia ou do tratamento (MARTIN, 2002).

A vulnerabilidade da criança afetada por um distúrbio do desenvolvimento sexual pode, ainda, ser agravada na medida da exposição ou do isolamento da criança quando ela se dá conta de suas diferenças em relação a seus irmãos ou a outras crianças No entanto, se aceita pela família em sua condição de DDS, poderá passar pela revelação à sociedade de maneira tranquila, segura e sem traumas (SPRIGGS; SAVULESCU, 1998).

Frader et al. (2004) apontam diretrizes como: adiamento da cirurgia de alteração de aparência até que a criança possa participar da decisão; necessidade de cuidados multidisciplinares aos pacientes e suas famílias; necessidade de estudos longitudinais rigorosos bem como a necessidade de treinamento adicional aos profissionais da saúde referente às condições de DDS como forma de minimizar os danos e riscos aos sujeitos DDS.

Bradley et al. (1998), por seu lado, mostram os conflitos psicossociais vivenciados por pessoas com indefinição da preferência sexual ao mesmo tempo em que traçam um paralelo entre os dois pacientes descritos no estudo para aceitar a sua condição; segundo os autores, foram fatores importantes a idade com que foi realizada a cirurgia e a menor ambivalência dos pais em relação à questão da sexualidade da criança. 
Finalmente, Maharaj et al. (2005) tecem considerações ético-legais da abordagem das crianças com DDS apontando para aconselhamento extenso e contínuo, consentimento informado, aderência a normas éticas e legais, abordagem multidisciplinar e a não adoção de abordagem paternalística.

\section{Consentimento}

Como em qualquer abordagem bioética envolvendo crianças e/ ou adolescentes, o termo de consentimento é ponto de tensão entre indivíduos, famílias, profissionais, eticistas e ativistas na abordagem e no tratamento das condições de distúrbios do desenvolvimento sexual, pois os pais ou responsáveis legais firmam o consentimento "no melhor interesse da criança" (HUGHES, 2007).

Alguns aspectos merecem considerações quando se pensa no consentimento. A literatura é vasta em opiniões conflitantes e em diretrizes que buscam minimizar os conflitos e tensões sobre essa questão.

O consentimento dos pais é exigido para menores de idade, exceto em casos de risco de morte. Uma questão limítrofe é quando o benefício é para o menor ou para uma terceira parte. Um argumento é que a cirurgia cosmética beneficia muito mais os pais do que as crianças nas fases iniciais. As ramificações éticas do consentimento informado e o direito dos pais de escolher ou decidir pela criança são particularmente ambíguas quando a doença ou a condição não apresenta risco de vida.

Os sujeitos portadores de DDS vêm questionando os profissionais que os atendem e se perguntando se as cirurgias e o curso de tratamento recomendado para esses indivíduos constituem consentimento informado (DREGER, 1999; MARTIN, 2002). E Martin (2002) ainda questiona o que constitui o consentimento informado quando os médicos tratam o indivíduo DDS e sua família. O mesmo autor comenta que consentimento informado é um tema de preocupação para os sujeitos DDS e afeta os pais, a criança DDS e as relações médico-pais, pais-criança, médico-paciente. O consentimento ainda é uma preocupação mesmo quando o padrão recomendado é seguido e há envolvimento de um psicólogo porque o curso de tratamento decidido frequentemente inclui esconder deliberadamente 
as informações médicas da criança. Segundo essa autora é importante revelar, na abordagem do sujeito e sua família, e no consentimento informado, o diagnóstico, a natureza e o propósito do tratamento, os riscos e os resultados, alternativas, prognóstico com e sem o tratamento e conflitos de interesse.

Coleman (2007), em seu estudo sobre os aspectos ético-legais da pesquisa em pediatria, cita a posição do teólogo e eticista Prof. Ramsey (2002 apud COLEMAN, 2007), o qual considera moralmente inaceitável o consentimento paterno para experiências perigosas, ou de outra categoria, que não tenham significado diagnóstico ou terapêutico para a criança. Coleman (2007) cita também McCormick (apud COLEMAN, 2007), o qual postula a validade do consentimento dos pais desde que este reflita uma presunção razoável do desejo da criança.

A alternativa que muitos profissionais apontam (e as organizações de apoio aos portadores de DDS sugerem) é que a criança/adolescente possa participar da tomada de decisões sobre sua vida. E então o questionamento que surge refere-se a qual seria a idade apropriada para o menor firmar assentimento ou consentimento ou o não assentimento, até que idade o menor é presumivelmente considerado incapaz de tomar decisões (KIPNIS, 2003).

Para Kipnis (2003), a regra dos 7 anos tem limitações, pois, nesta idade, o menor ainda é presumivelmente considerado incapaz de tomar decisões e essa presunção não pode ser superada; após os 7 anos, a capacidade de tomar decisão pode ser avaliada no contexto da conversa educativa; dos 7 aos 14 anos, a incapacidade presumida pode ser relevada em determinadas situações, dependendo da capacidade da criança em entender o problema médico e as alternativas e sua capacidade de deliberar sobre o tema; para crianças com mais de 14 anos, existe uma capacidade presumida de tomar decisões. Todavia, cada caso é um caso, e o profissional da saúde deve avaliar a capacidade de decisão do menor de 14 anos e a incapacidade de tomar decisões de um sujeito maior de 14 anos, pois a capacidade decisória não está apenas relacionada com a idade ou com o status legal (KIPNIS, 2003).

Já em Levy, Larcher e Kurz (2003), encontra-se que o consentimento ou assentimento é uma exigência para todos os aspectos dos cuidados 
médicos, para medidas preventivas, diagnósticas ou terapêuticas e para a pesquisa. Segundo esses autores, as crianças podem, efetivamente, recusar o tratamento ou procedimentos que não sejam necessários para salvar suas vidas ou prevenir danos graves. Comentam ainda a Convenção das Nações Unidas dos Direitos da Criança, a qual assegura mecanismos para que a criança, com menos de 18 anos seja ouvida, por considerá-la como pessoa, ainda que menor de idade.

Wieseman et al. (2010), ao abordarem a questão da autonomia do sujeito DDS apontam para a moratória à cirurgia precoce (a menos que haja emergência médica) e estabelecem três princípios éticos importantes da abordagem do sujeito DDS, centrados no bem estar da criança e do futuro adulto, na defesa dos direitos da criança e no respeito à família e às relações entre pais e filhos. Para esses autores, a criança deve participar ativamente das decisões sobre sua condição, pois consideram que elas são aptas e inclusive têm direito a veto. No entanto, também mostram claramente as incongruências de se estabelecerem idades limites para o assentimento/consentimento da criança face à decisões tão graves e irreversíveis.

Diamond (2004) também apresenta três recomendações básicas, estabelecidas pela Associação Americana de Pediatria, que se aplicam a psiquiatras e pediatras. Na Conferência do Texas, realizada em 1999, foi reforçada a necessidade de mais investigações e estudos de longo termo bem como a obrigatoriedade da revelação de sua condição à criança e o terceiro tema que ressurgiu foi o da necessidade de reconhecer o cérebro como um órgão sexual (DIAMOND, 2004).

Como resultado da complexidade das condições DDS, dos conflitos e dos dilemas apresentados pela tomada de decisões referentes à reatribuição de sexo, foram propostos três modelos de termo de consentimento, descritos em Martin (2002). Apresentamos a seguir resumidamente tais modelos: o Modelo Colombiano preconiza um consentimento qualificado e consistente, limitando a autoridade paterna à exigência e urgência do procedimento, à invasibilidade e à idade e autonomia da criança. Com esse modelo, os pais não podem consentir por crianças com mais de 5 anos, pois estas crianças são autônomas e já se identificaram com o gênero; o modelo ISNA, dentre outras argumentações, considera as cirurgias 
realizadas na infância como mutilação genital do intersexo; e o modelo Diamond postula que o consentimento deve ser uma revelação honesta e completa da condição, para os pais e para a criança, de acordo com a idade. Esse modelo recomenda que conste do consentimento a diferença entre cirurgia estética e aquela necessária para manter a saúde física e mental, enfatizando a funcionalidade sobre a aparência. Os pais devem ser informados da impossibilidade de obter o consentimento antes da puberdade; das implicações decorrentes de se esperar até lá e também devem estar cientes dos efeitos e repercussões do tratamento com hormônio, além de considerar o momento em que a criança deve ser informada da cirurgia (MARTIN, 2002).

Diamond et al. (2006), em estudo envolvendo questionário aplicado a profissionais pediatras que atendem crianças com DDS, mostram as controvérsias e dificuldades na tomada de decisão e na abordagem dos pacientes DDS.

Kipnis (2004) comenta que o psiquiatra da infância e da adolescência se defronta com três aspectos delicados da abordagem dos DDS: as responsabilidades éticas para com sujeitos de menor idade; o sujeito/ paciente menor e seus pais.

\section{Conclusão}

A identidade sexual interfere com todos os aspectos da vida em sociedade que demandam tal identidade. Consequentemente, os DDSs configuram condições que geram vulnerabilidade, conflitos, e a escolha da melhor abordagem das crianças com DDS é tarefa árdua, pois são muitas as decisões importantes de alta complexidade e irreversíveis a serem tomadas, e que estão relacionadas com sexo de criação, cirurgias, reposição hormonal e aspectos psicossociais.

Confidencialidade e consentimento informado traduzem o respeito ao sujeito e à sua autonomia e, ao mesmo tempo, buscam salvaguardar os direitos e proteger o sujeito e o profissional da saúde. A decisão judicial da Colômbia vem desafiando a prática de cirurgia em indivíduos com DDS em seu mais alto nível e pode refletir em implicações nos direitos humanos e de crianças. 
Considerar a criança como pessoa e ser dotado de direitos são deveres dos profissionais, pois as escolhas são difíceis, e outorgar a outrem a definição do sexo da criança, sem que ela possa opinar, fere os princípios de justiça, autonomia e beneficência. Postergar a cirurgia, até que a criança possa participar da tomada de decisões, certamente também tem suas implicações no bem-estar da criança; no entanto só ela pode escolher, no momento certo, e ainda assim, com risco de que, na vida adulta não se sinta feliz com a decisão tomada na infância.

Dos paradigmas paternalistas migramos para o atendimento focado no sujeito, para a consideração da tomada de termo de consentimento/assentimento do sujeito DDS e quem sabe chegaremos ao consenso de que distúrbios do desenvolvimento sexual são apenas mais uma variabilidade genética. Profissionais de saúde treinados e trabalhando a multidisciplinaridade do atendimento aos pacientes com DDS, constitui uma conduta mais próxima do ideal para a abordagem adequada dessas condições.

Dada a complexidade e a delicadeza de cada situação, e a irreversibilidade do procedimento, todos os casos de DDS demandam cuidado extremo na abordagem e no tratamento, e requerem acompanhamento multidisciplinar e de longo termo. A minimização dos desconfortos, riscos e insatisfações e das vulnerabilidades somente será alcançada com um atendimento centrado no paciente, respeitando sua autonomia, suas ansiedades e seus temores, analisando cada caso individualmente e com a participação de todos - profissionais da saúde, família e do sujeito afetado pelo DDS.

\section{Referências}

ALLEN, L. Disorders of sexual development. Obstetrics \& Gynecology Clinics of North America, v. 36, n. 1, p. 25-45, 2009.

BARBARO, M.; WEDELL, A.; NORDENSTRÖM, A. Disorders of sex development. Seminars in Fetal and Neonatal Medicine, v. 16, n. 2, p. 119-127, 2011. 
BARTHOLD, J. S. Disorders of sex differentiation: a pediatric urologist's perspective of new terminology and recommendations. The Journal of Urology, v. 185, n. 2, p. 393-400, 2011.

BRADLEY, S. J. et al. Experiment of nurture: ablatio penis at 2 months, sex reassignment at 7 months, and a psychosexual follow-up in young adulthood. Pediatrics, v. 102, n. 1, p. e9, 1998.

COLEMAN, D. L. The legal ethics of pediatric research. Duke Law Journal, v. 57, n. 3, p. 517-524, 2007.

DAABOUL, J.; FRADER, J. Ethics and the management of the patient with intersex: a middle way. Journal of Pediatric Endocrinology \& Metabolism, v. 14, n. 9, p. 1575-1583, 2001.

DIAMOND, M. A critical evaluation of the ontogeny of human sexual behavior. 1965. Disponível em: <http://www.hawaii.edu/PCSS/biblio/articles/ 1961to1999/1965-critical-evaluation.html>. Acesso em: 12 jun. 2011.

DIAMOND, M. Sex, gender, and identity over the years: a changing perspective. Child \& Adolescent Psychiatric Clinics of North America, v. 13, p. 591-607, 2004.

DIAMOND, D. A. et al. Sex assignment for newborns with ambiguous genitalia and exposure to fetal testosterone: attitudes and practices of pediatric urologists. Journal of Pediatrics, v. 148, n. 4, p. 445-449, 2006.

DREGER, A. M. (Ed.). Intersex in the age of ethics. Hagerstown: University Publishing Group, 1999.

FRADER, J. et al. Health care professionals and intersex conditions. Archives of Pediatric \& Adolescent Medicine, v. 158, n. 5, p. 426-428, 2004.

GILLAM, L. H.; HEWITT, J. K.; WARNE, G. L. Ethical principles for the management of infants with disorders of sexual development. Hormone Residence in Paediatrics, v. 74, n. 6, p. 412-418, 2010.

HUGHES, I. A. et al. Consensus statement on management of intersex disorders. Pediatrics, v. 118, n. 2, p. 448-500, 2006. 
HUGHES, I. A. et al. Consequences of the ESPE/LWPES guidelines for diagnosis and treatment of disorders of sex development. Best Practice \& Research Clinical Endocrinology \& Metabolism, v. 21, n. 3, p. 351-365, 2007.

KIPNIS, K. Seven vulnerabilities in pediatric research. Theoretical Medicine, v. 24, n. 2, p. 1076-1120, 2003.

KIPNIS, K. Gender, sex, and professional ethics in child and adolescent psychiatry. Child \& Adolescent Psychiatric Clinics of North America, v. 13, n. 3, p. 695-708, 2004.

LEVY, M. L.; LARCHER, V.; KURZ, R. Informed consent/assent in children. Statement of the ethics working group of the Confederation of European Specialists in Pediatrics. European Journal of Pediatrics, v. 162, n. 9, p. 629-633, 2003.

LUX, A. et al. Clinical evaluation study of the german network of disorders of sex development (DSD)/intersexuality: study design, description of the study population, and data quality. BMC Public Health, v. 9, p. 110, 2009.

MAHARAJ, N. R. et al. Intersex conditions in children and adolescents: surgical, ethical and legal considerations. Journal of Pediatric and Adolescent Gynecology, v. 18, n. 6, p. 399-402, 2005.

MARTIN, P. Moving toward an international standard in informed consent: the impact of intersexuality and the internet on the standard of care. Duke Journal of Gender Law \& Policy, v. 9, n. 135, p. 135-169, 2002.

MIESZCZAK, J.; HOUK, C. P.; LEE, P. A. Assignment of the sex of rearing in the neonate with a disorder of sex development. Current Opinion in Pediatrics, v. 21, n. 4, p. 541-547, 2009.

PASTERNAK, J. Genética molecular humana: mecanismos das doenças hereditárias. São Paulo: Manole, 2002.

RANGECROFT, L. Surgical management of ambiguous genitalia. Archives of Disease in Childhood, v. 88, n. 9, p. 799-801, 2003.

REY, R. A.; GRINSPON, R. P. Normal male sexual differentiation and aetiology of disorders of sex development. Best Practice \& Research Clinical Endocrinology \& Metabolism, v. 25, n. 2, p. 221-238, 2011. 
SPRIGGS, M.; SAVULESCU, J. The ethics of surgically assigning sex to intersex infants. In: BENATAR, D. (Ed.). Cutting to the core: exploring the ethics of contested surgeries. Lanham: Rowman \& Littlefield Publishers, 1998. p. 638-658.

WALKER, J. The death of David Reimer: a tale of sex, science and abuse. 2004. Disponivel em: <http://reason.com/archives/2004/05/24/the-death-of-david-reimer > Acesso em: 9 June 2011.

WIESEMANN, C. et al. Ethical principles and recommendations for the medical management of differences of sex development (DSD)/intersex in children and adolescents. European Journal of Pediatrics, v. 169, n. 6, p. 671-679, 2010.

Recebido: 15/06/2011

Received: 06/15/2011

Aprovado: 06/10/2011

Approved: 10/06/2011 